\title{
Electrochemical Triggered Open and Closed Pacman Bis-metalloporphyrins
}

Grégory Pognon ${ }^{\mathrm{a}}$ Corinne Boudon ${ }^{\mathrm{b}}$ Kurt J. Schenk, ${ }^{\mathrm{c}}$ Michel Bonin, ${ }^{\mathrm{c}}$ Benoit Bach, ${ }^{\mathrm{b}}$ and Jean

$$
\text { Weiss }^{\text {a* }} \text {. }
$$

a) CLAC, and b) Laboratoire d'Electrochimie, Institut de Chimie LC3 UMR 7177 CNRS-ULP, 4, rue Blaise Pascal, 67070 Strasbourg, France; c) ÉPFL, LCr1-IPMC-FSB, BSP Dorigny, CH1015 Lausanne, Switzerland

\section{Supporting Information}

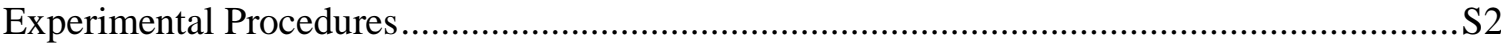

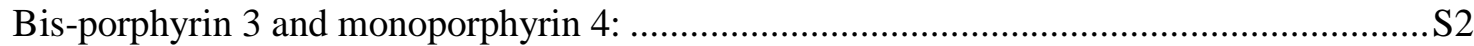

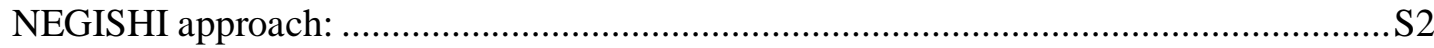

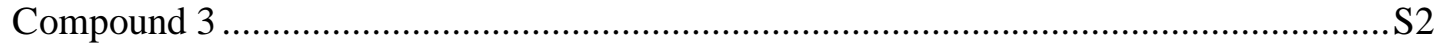

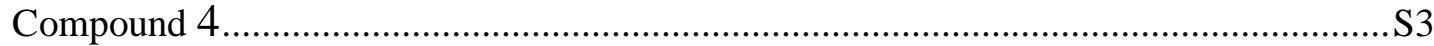

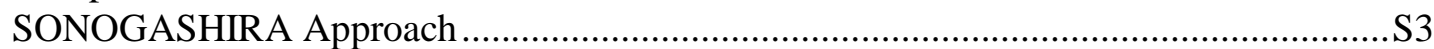

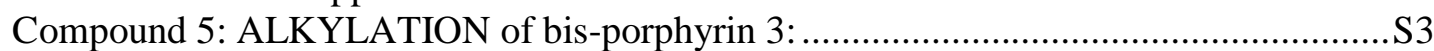

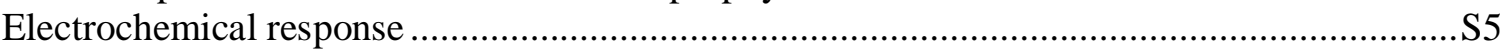

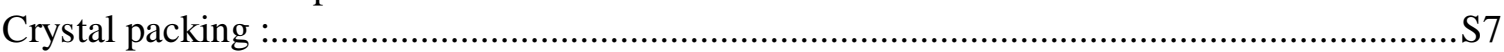

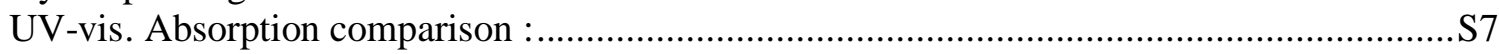




\section{Experimental Procedures}

\section{Bis-porphyrin 3 and monoporphyrin 4:}

\section{NEGISHI approach:}

In a flask thoroughly flushed with argon, $\mathrm{Et}_{3} \mathrm{~N}(1 \mathrm{ml}, 7.2 \mathrm{mmol})$ was added to a solution of $\mathrm{ZnBr}_{2}(37 \mathrm{mg}, 0.162 \mathrm{mmol})$ in $10 \mathrm{ml}$ of freshly distilled THF. The reaction mixture (degassed) turned pale pink after stirring for $1-3 \mathrm{~min}$ at $25^{\circ} \mathrm{C}$. The reaction mixture was then successively treated (and degassed) with the diiodo-crown[6]-dihydroxycalix[4]arene 1 (62 $\mathrm{mg}, 0.071$ mmol), meso ethynyl-NiOEP 2 (100 mg, $0.162 \mathrm{mmol})$, and $\mathrm{Pd}\left(\mathrm{PPh}_{3}\right)_{4}(10 \mathrm{mg}, 0.008 \mathrm{mmol})$. The reaction was TLC monitored using silica gel plates and cyclohexane/ $\mathrm{CH}_{2} \mathrm{Cl}_{2}$ and $\mathrm{CH}_{2} \mathrm{Cl}_{2}$ /acetone (80/20) mixtures as eluants. After 3 days at $55^{\circ} \mathrm{C}, \mathrm{Pd}\left(\mathrm{PPh}_{3}\right)_{4}(10 \mathrm{mg})$ and $\mathrm{ZnBr}_{2}(37 \mathrm{mg})$ were added. After 7 days, the solvent was evaporated, the residue dissolved in $\mathrm{CH}_{2} \mathrm{Cl}_{2}$, washed with aqueous $\mathrm{NH}_{4} \mathrm{Cl}, \mathrm{HCl}(10 \%)$, aqueous $\mathrm{NaHCO}_{3}, \mathrm{HCl}(10 \%), \mathrm{H}_{2} \mathrm{O}$ (twice) and then dried by 3 azeotropic evaporations (toluene-ethanol). The crude mixture was purified by column chromatography $\left(\mathrm{SiO}_{2}, \mathrm{CH}_{2} \mathrm{Cl}_{2}\right)$ to eliminate starting material (ethynylporphyrin) and butadiyne products (degradation of ethynylporphyrin). A polarity gradient was then introduced using increasing amounts of acetone in $\mathrm{CH}_{2} \mathrm{Cl}_{2}(2.5 ; 5 ; 7.5 ; 10$; $15 ; 20 ; 25 \%)$ to collect, in two consecutive red bands, the bisporphyrinic and monoporphyrinic adducts. Pure compounds were obtained by recrystallisation in a mixture $\mathrm{CH}_{2} \mathrm{Cl}_{2} / \mathrm{MeOH}$ (bisporphyrinic : $58 \mathrm{mg}, 0.031 \mathrm{mmol}, 45 \%$; monoporphyrinic : $15 \mathrm{mg}, 0.011$ mmol, $15 \%)$.

\section{Compound 3}

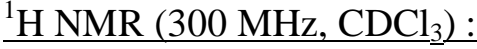

$9.41\left(\mathrm{~s}, 4 \mathrm{H}, \mathrm{H}_{\text {meso }}\right), 9.38$ (s, 2H, $\left.\mathrm{H}_{\text {meso }}\right), 8.13$ (s, 2H, OH), 7.48 (s, 4H, $\mathrm{H}_{\text {meta(porph) }}$ ), 7.01 (d, $\left.\mathrm{J}=7.3 \mathrm{~Hz}, 4 \mathrm{H}, \mathrm{H}_{\text {meta }(\mathrm{H})}\right), 6.87\left(\mathrm{t}, \mathrm{J}=7.3 \mathrm{~Hz}, 4 \mathrm{H}, \mathrm{H}_{\mathrm{para}}\right), 4.50\left(\mathrm{~d}, \mathrm{~J}=13 \mathrm{~Hz}, 4 \mathrm{H}, \operatorname{ArCH}_{2} \mathrm{Ar}\right), 4.25$ (q, $\left.\mathrm{J}=7.1 \mathrm{~Hz}, 12 \mathrm{H} \mathrm{C}_{2} \mathrm{CH}_{3}\right), 4.05\left(\mathrm{t}, \mathrm{J}=3.8 \mathrm{~Hz}, 4 \mathrm{H}, \mathrm{H}_{\text {crown }}, 3.95\right.$ (t, J=3.7Hz, $\left.4 \mathrm{H}, \mathrm{H}_{\text {crown }}\right), 3.88$ (t, $\left.\mathrm{J}=4 \mathrm{~Hz}, 4 \mathrm{H}, \mathrm{H}_{\text {crown }}\right), 3.78\left(\mathrm{t}, \mathrm{J}=7.5 \mathrm{~Hz}, 26 \mathrm{H}, \mathrm{H}_{\text {crown }}+\mathrm{C}_{2} \mathrm{CH}_{3}+\mathrm{s}, 4 \mathrm{H}, \mathrm{H}_{\text {crown }}\right), 3.49$ (m (q+d), $8 \mathrm{H}$, $\left.\mathrm{C}_{2} \mathrm{CH}_{3}+\mathrm{ArCH}_{2} \mathrm{Ar}\right), 1.84\left(\mathrm{t}, \mathrm{J}=7.3 \mathrm{~Hz}, 12 \mathrm{H}, \mathrm{CH}_{2} \underline{\mathrm{C}}_{3}\right) 1.74\left(\mathrm{~m}(\mathrm{t}+\mathrm{t}), \mathrm{J}=7.3 \mathrm{~Hz}, 36 \mathrm{H}, \mathrm{CH}_{2} \mathrm{CH}_{3}\right)$.

$\underline{\mathrm{UV}-\mathrm{Vis}}\left(\lambda_{\max }\right.$ in $\mathrm{nm}\left(\varepsilon\right.$ in $\left.\left.\mathrm{M}^{-1} . \mathrm{cm}^{-1}\right)\right)$ :

Toluene : 435 (220656), 554 (15956), 589 (15656)

$\mathrm{CH}_{2} \mathrm{Cl}_{2}: 433$ (224387.5), 551 (17816), 583 (17191)

DMF : 435 (211691), 551 (14205), 583 (13684)

$\underline{\operatorname{MS}(\mathrm{M} / \mathrm{e})}: \mathrm{C}_{114} \mathrm{H}_{126} \mathrm{~N}_{8} \mathrm{Ni}_{2} \mathrm{O}_{8}: 1852.8$ (calcd : 1853.66) 


\section{Compound 4}

${ }^{1} \mathrm{H}$ NMR (300 MHz, CDCl3) :

$9.43\left(\mathrm{~s}, 2 \mathrm{H}, \mathrm{H}_{\text {meso }}\right), 9.39$ (s, 1H, $\left.\mathrm{H}_{\text {meso }}\right), 7.96(\mathrm{~s}, 1 \mathrm{H}, \mathrm{OH}), 7.77(\mathrm{~s}, 1 \mathrm{H}, \mathrm{OH}), 7.47(\mathrm{~s}, 2 \mathrm{H}$, $\left.\mathrm{H}_{\text {meta(porph) }}\right), 7.38$ (s, 2H, $\left.\mathrm{H}_{\text {meta(I) }}\right), 6.96$ (d, J=7.3, 2H, $\left.\mathrm{H}_{\text {meta(H) }}\right), 6.89$ (d, J=6.4, 2H, $\left.\mathrm{H}_{\text {meta(H) }}\right), 6.81$ (t, J=7.5, 2H, $\mathrm{H}_{\text {para }}$ ), 4.45 (d, J=13.2, 2H, $\mathrm{ArCH}_{2} \mathrm{Ar}$ ), 4.37 (d, J=13.2, 2H, $\mathrm{ArCH}_{2} \mathrm{Ar}$ ), 4.26 (q, $\left.\mathrm{J}=7.1,4 \mathrm{H}, \mathrm{C}_{2} \mathrm{CH}_{3}\right), 4.14\left(\mathrm{~m}, 4 \mathrm{H}, \mathrm{H}_{\text {crown }}\right) 3.99\left(\mathrm{~m}, 4 \mathrm{H}, \mathrm{H}_{\text {crown }}\right), 3.90\left(\mathrm{~m}, 4 \mathrm{H}, \mathrm{H}_{\text {crown }}\right), 3.82(\mathrm{~m}$, $\left.16 \mathrm{H}, \mathrm{H}_{\text {crown }}+\mathrm{C}_{2} \mathrm{CH}_{3}\right), 3.71$ (s, $\left.4 \mathrm{H}, \mathrm{H}_{\text {crown }}\right), 3.45$ (d, J=13.2, $2 \mathrm{H}, \mathrm{ArCH}_{2} \mathrm{Ar}$ ), 3.31 (d, J=13.2, $\left.2 \mathrm{H}, \mathrm{ArCH}_{2} \mathrm{Ar}\right), 1.85$ (t, J=7.1, 6H, $\left.\mathrm{CH}_{2} \mathrm{C}_{3}\right), 1.77\left(\mathrm{~m}, 18 \mathrm{H}, \mathrm{CH}_{2} \mathrm{CH}_{3}\right)$.

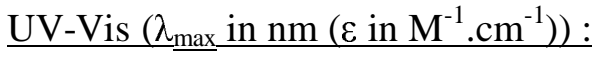

Toluene : 433 (154039), 554 (10253), 589 (10188)

$\mathrm{CH}_{2} \mathrm{Cl}_{2}: 434$ (168433), 553 (21420), 587 (20026)

DMF : 435 (152086), 554 (11136), 583 (10774)

\section{SONOGASHIRA Approach}

In a flask thoroughly flushed with argon, porphyrin $2(140 \mathrm{mg}, 0.227 \mathrm{mmol})$ and calixaren 1 (91 mg, $0.103 \mathrm{mmol})$ were added to a degassed mixture of distilled toluene/ $\mathrm{Et}_{3} \mathrm{~N}(50 / 50 ; 20$ $\mathrm{ml})$, and then catalytic amounts of $\mathrm{Pd}\left(\mathrm{PPh}_{3}\right)_{2} \mathrm{Cl}_{2}$ and $\mathrm{CuI}(10 \%$ mol.). The degassed reaction mixture was stirred at $55{ }^{\circ} \mathrm{C}$ for $48 \mathrm{~h}$. The solvents were evaporated, the residue dissolved in $\mathrm{CH}_{2} \mathrm{Cl}_{2}$, washed 3 times with water, and dried by 3 azeotropic evaporation (toluene-ethanol). The crude mixture was purified by column chromatography $\left(\mathrm{SiO}_{2}, \mathrm{CH}_{2} \mathrm{Cl}_{2}\right)$ to eliminate starting material (ethynylporphyrin) and diporphyrin butadiyne by-product (homo-coupling) and others degradations products. A polarity gradient was then introduced with increasing proportions of acetone in $\mathrm{CH}_{2} \mathrm{Cl}_{2}(2.5 ; 5 ; 7.5 ; 10 ; 15 ; 20 ; 25 \%)$ to collect, in two consecutive red bands, bisporphyrinic and monoporphyrinic products 3 and $\mathbf{4}$. Pure compounds were obtained by recrystallization in a $\mathrm{CH}_{2} \mathrm{Cl}_{2} / \mathrm{MeOH}$ mixture (bisporphyrinic : $50 \mathrm{mg}$, $0.027 \mathrm{mmol}, 25 \%$; monoporphyrinic : $20 \mathrm{mg}, 0.015 \mathrm{mmol}, 15 \%$ ).

\section{Compound 5: ALKYLATION of bis-porphyrin 3:}

In a flask thoroughly flushed with argon, $\mathrm{NaH}(10 \mathrm{mg}, 0.4 \mathrm{mmol})$ was added to a degassed solution of bisporphyrinic derivative $3(70 \mathrm{mg}, 0.038 \mathrm{mmol})$ in $20 \mathrm{ml}$ of freshly distilled THF. The reaction mixture was stirred at $55{ }^{\circ} \mathrm{C}$ for $30-45 \mathrm{~min}$. Then propyl bromide $(41 \mathrm{mg}, 0.35$ mmol) and $\mathrm{NaI}$ were added. The reaction by TLC using silica gel plates and a $\mathrm{CH}_{2} \mathrm{Cl}_{2} /$ acetone (75/25) mixture as eluant. Two products were formed : mono and dipropoxy-calixaren ; after 3 days supplementary $\mathrm{NaH}$ was added $(10 \mathrm{mg}, 0.4 \mathrm{mmol})$. After 7 days the reaction was stopped and quenched with $25 \mathrm{ml}$ of $10 \% \mathrm{HCl}$. The solvent was evaporated and the residue dissolved in $\mathrm{CH}_{2} \mathrm{Cl}_{2}$. The organic phase was washed three times with water and dried by three successive azeotropic evaporations mixture (toluene-ethanol). The exact same alkylation procedure was repeated using the monopropoxy and dipropoxy derivatives mixture to finally obtain the 
dipropoxy derivative as major product. Direct addition of extra propyl bromide or $\mathrm{NaH} / \mathrm{NaI}$ to the alkylation reaction gave considerably worse results. The residue was then purified by column chromatography $\left(\mathrm{SiO}_{2}, \mathrm{CH}_{2} \mathrm{Cl}_{2}\right.$ /Acetone $(95 / 5)$ and $\left.\mathrm{SiO}_{2}, \mathrm{CH}_{2} \mathrm{Cl}_{2}\right)$ to afford $\mathbf{5}$ as a purple solid (40 mg, $0.021 \mathrm{mmol}, 55 \%$ )

\section{$\underline{{ }^{1} \mathrm{H} \text { NMR (300 MHz, CDCl3) : }}$}

$8.53\left(\mathrm{~s}, 2 \mathrm{H}, \mathrm{H}_{\text {meso }}\right), 8.23\left(\mathrm{~s}, 4 \mathrm{H}, \mathrm{H}_{\text {meso }}\right), 7.32\left(\mathrm{~d}, \mathrm{~J}=7.5 \mathrm{~Hz}, 4 \mathrm{H}, \mathrm{H}_{\text {meta( } \mathrm{H})}\right), 7.15(\mathrm{t}, \mathrm{J}=7.5 \mathrm{~Hz}, 2 \mathrm{H}$, $\left.\mathrm{H}_{\text {para }}\right), 6.85\left(\mathrm{~s}, 4 \mathrm{H}, \mathrm{H}_{\text {meta(porph) }}\right), 4.56\left(\mathrm{~d}, \mathrm{~J}=13.2 \mathrm{~Hz}, 4 \mathrm{H}, \mathrm{ArCH}_{2} \mathrm{Ar}\right), 4.43$ (pseudo $\mathrm{q}, 4 \mathrm{H}$, $\mathrm{CH}_{2} \mathrm{CH}_{3}$ ), 4.31 (pseudo q, 2H, $\mathrm{CH}_{2} \mathrm{CH}_{3}$ ), 4.19 (pseudo q, 6H, $\underline{\mathrm{H}}_{2} \mathrm{CH}_{3}$ ), 3.82 (m, 26H, $\left.\mathrm{C}_{2} \mathrm{CH}_{3}+\mathrm{H}_{\text {crown }}\right), 3.35$ (d, J=13.2, $\left.4 \mathrm{H}, \mathrm{ArCH}_{2} \mathrm{Ar}\right), 3.25\left(\mathrm{~m}, 16 \mathrm{H}, \mathrm{C}_{2} \mathrm{CH}_{3}+\mathrm{OCH}_{2} \underline{\mathrm{C}}_{2} \mathrm{CH}_{3}\right)$, $2.91\left(\mathrm{~m}, 8 \mathrm{H}, \mathrm{OC}_{2} \mathrm{CH}_{2} \mathrm{CH}_{3}+\mathrm{C}_{2} \mathrm{CH}_{3}\right), 2.03\left(\mathrm{q}, \mathrm{J}=7.3 \mathrm{~Hz}, 6 \mathrm{H}, \mathrm{OCH}_{2} \mathrm{CH}_{2} \mathrm{CH}_{3}\right), 1.75(\mathrm{~m}, 4 \mathrm{H}$, $\left.\mathrm{CH}_{2} \mathrm{CH}_{3}\right), 1.54\left(\mathrm{t}, \mathrm{J}=7.5 \mathrm{~Hz}, 18 \mathrm{H}, \mathrm{CH}_{2} \underline{\mathrm{C}}_{3}\right), 1.38\left(\mathrm{~m}, 18 \mathrm{H}, \mathrm{CH}_{2} \underline{\mathrm{C}}_{3}\right), 1.14(\mathrm{t}, \mathrm{J}=7.5 \mathrm{~Hz}, 8 \mathrm{H}$, $\left.\mathrm{CH}_{2} \underline{\mathrm{CH}}_{3}\right)$.

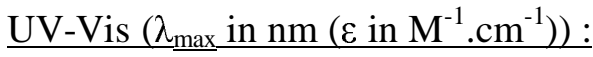

Toluene : 419 (164469), 552 (18428), 593 (19031)

$\mathrm{CH}_{2} \mathrm{Cl}_{2}: 418$ (257978), 555 (17609), 594 (17587)

DMF : 419 (251441), 554 (14407), 594 (14197)

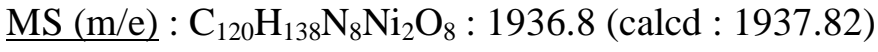

Analysis for : $\mathrm{C}_{120} \mathrm{H}_{138} \mathrm{~N}_{8} \mathrm{Ni}_{2} \mathrm{O}_{8}+1 / 2 \mathrm{CHCl}_{3}+1 / 2 \mathrm{CH}_{2} \mathrm{Cl}_{2}$

Calcd : $\quad \mathrm{C}: 71.04, \mathrm{H}: 6.79, \mathrm{~N}: 5.57$

Found : $\quad$ C : $70.98, \mathrm{H}: 6.93, \mathrm{~N}: 5.59$ 

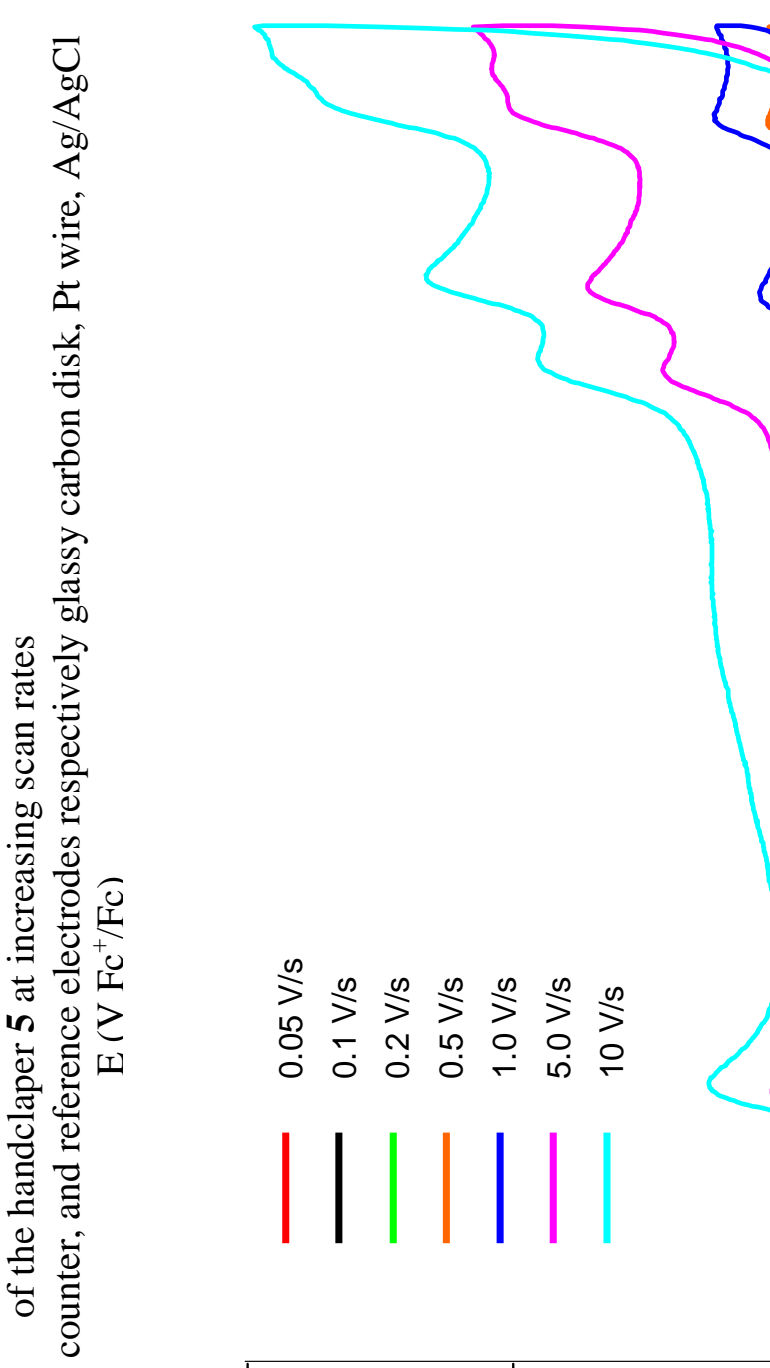

.
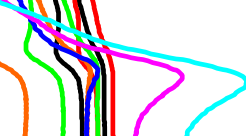

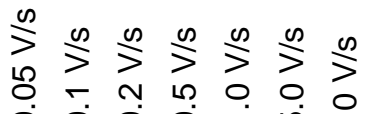

○்
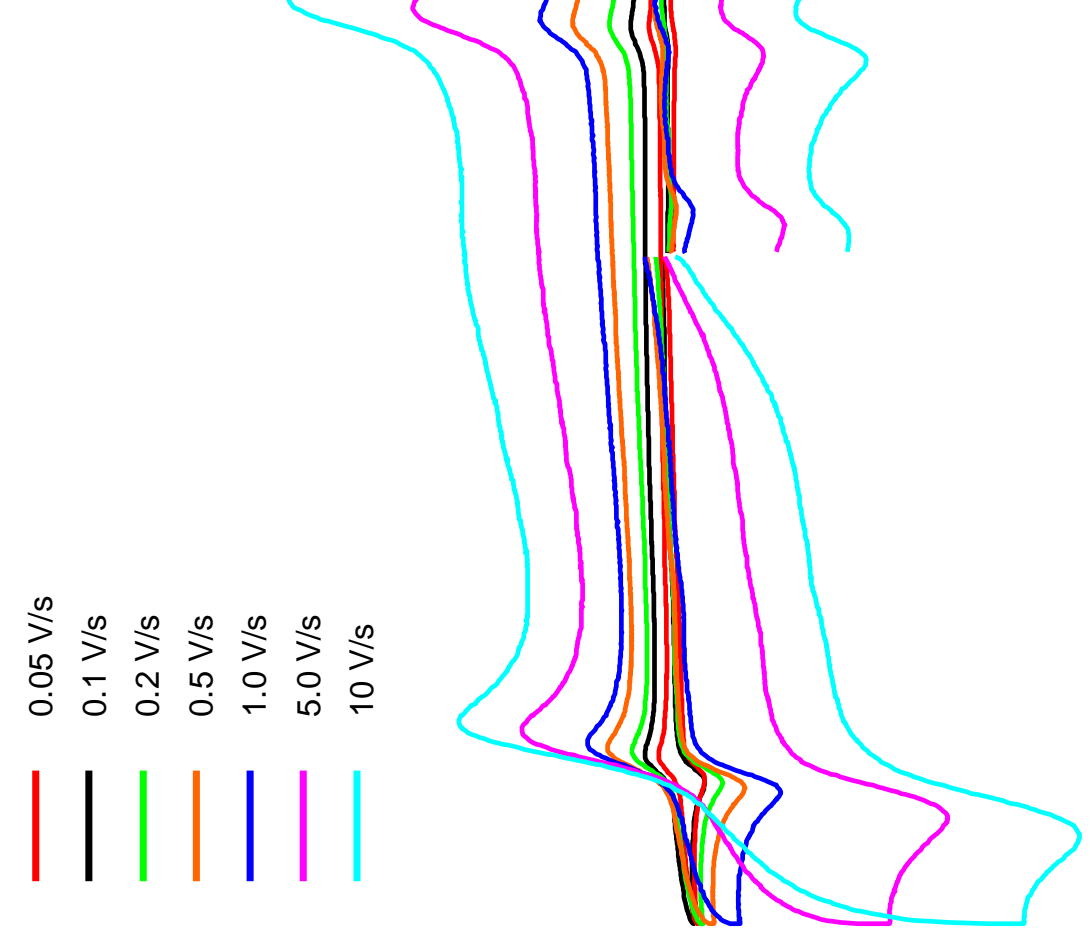

N

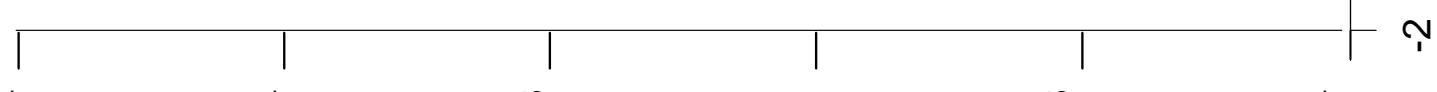

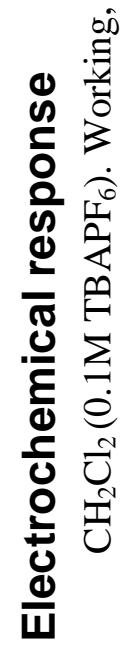



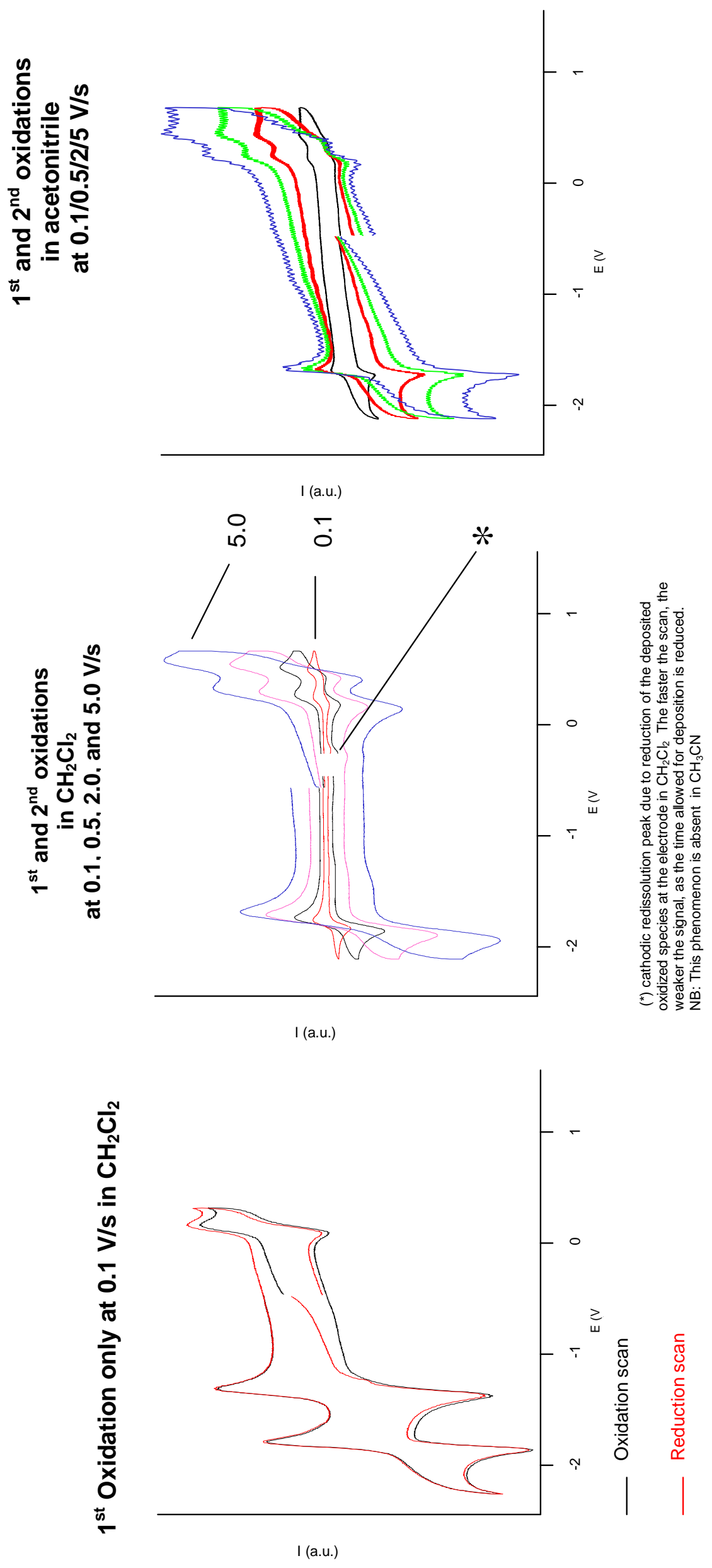


\section{Crystal packing :}

display of the unit cell.

(cyclohexane molecules (4) omitted for clarity)

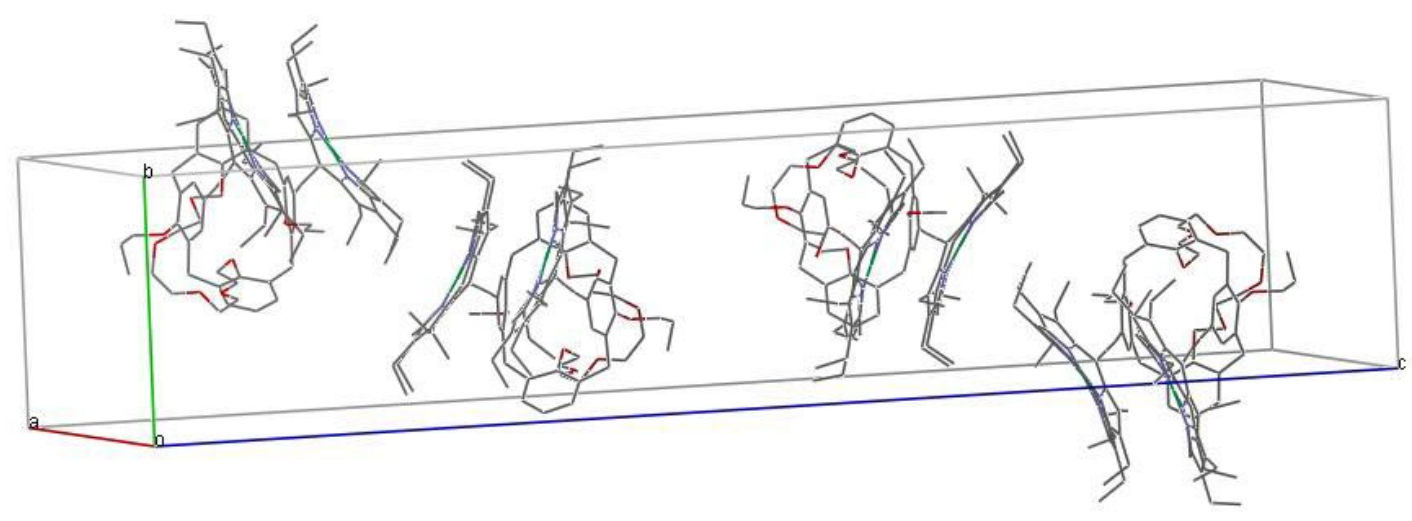

\section{UV-vis. Absorption comparison :}

for NiOEP and 5 in $\mathrm{CH}_{2} \mathrm{Cl}_{2}$. The broadening of the Soret band is significant of the excitonic coupling in a face-to-face geometry. The global red shift of the absorption is a consequence of the full conjugation of the porphyrin macrocycles with the phenyl rings of the calixarene platform through the ethynyl linker.

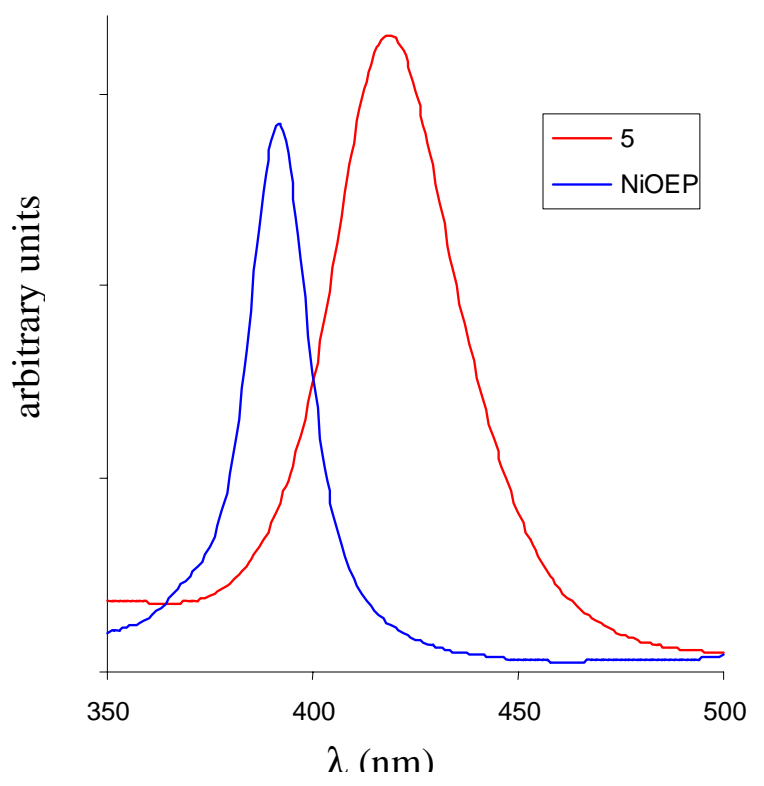

